\title{
Research on Cultural Influence Based on the Immersive Entertainment IP Experience Project
}

\author{
Mao Wang* and Zhucui Jing \\ School of Economics and Management, Beijing Jiaotong University, China, 100044 \\ *Corresponding author. Email: maom_idyllic@163.com
}

\begin{abstract}
With the continuous integration of culture and technology, new forms of cultural industry continue to emerge. As the intelligent product of the new era, the immersive entertainment IP experience project can not only reflect IP value with novel and innovative forms, but also enable participants to obtain high-quality personalized experience and spiritual satisfaction, and its cultural influence plays an increasingly important role in the construction of cultural display ability and cultural construction in China. At present, immersive entertainment IP experience project is still in the initial stage of development, so it is urgent to further study in expanding its popularity and improving its cultural influence to grasp the future development direction. This paper describes the immersive entertainment IP experience project, and makes an empirical analysis on its cultural influence. The research uses the method of questionnaire survey to collect the public awareness, participation and cultural influence evaluation of the immersive entertainment IP experience project. A total of 224 valid questionnaires were obtained. Then the paper uses regression analysis to get results about the effect of each dimension on cultural influence. Finally, combined with the research conclusion, the paper provides some meaningful suggestions, such as improving the overall quality of the project, creating a project integration platform, deepening the connection with Chinese traditional culture and enhancing social attention, so as to provide reference for promoting the high-quality development of immersive entertainment industry.
\end{abstract}

Keywords: Immersive entertainment IP experience project, Cultural influence, Public awareness.

\section{INTRODUCTION}

Nowadays, promoting the organic integration of culture and science and technology has become an important direction of high-quality and vigorous development of cultural industry. Under such circumstances, the immersion industry, as the wisdom product of the new era, its immersion injection makes the traditional mode have a huge subversion. This innovation model has been the attention of all walks of life and policy support. According to the White Paper on the Development of China's Immersion Industry in 2020[1], the total output value of global immersion industry has reached $\$ 5.19$ billion in 2019 , and the number of global immersive experience projects has reached as high as 8058, covering 12 major sub industries and 258 immersive formats. In addition, the total output value of China's immersion industry has reached 4.82 billion yuan in 2019, including exhibition, live entertainment, commercial real estate, cultural tourism and other related industries are paying close attention to the development of immersion industry with unprecedented enthusiasm.

As one of the branches of the huge immersion industry, participating in immersive experience has become an offline entertainment form that young consumers are flocking to. Immersive entertainment IP experience project is a form of entertainment that emphasizes experience and high participation. It uses optic artistry and equipment to package the immersive entertainment IP, and creates specific scenes to set off the theme, so that participants can mobilize multiple senses to participate in it and have interaction. For consumers and participants, the immersive entertainment project is a kind of enjoyment so that they can participate in it wholeheartedly and ignore the entertainment consumption, and then focus on the immersion and interaction, so as to obtain spiritual comfort and find the experience value. For creators and performers, they will face the test of innovation, and have a sense of achievement and gain that the creative value of the project is recognized. From the perspective 
of both sides, it is of great benefit and can naturally win the hearts of the people. As one of the emerging cultural projects, the immersive entertainment IP experience project has a profound impact on the cultural scope and effect.

There are various kinds of immersive entertainment IP experience projects, which can be divided into two categories according to the different emphasis of offline immersive entertainment -- One is based on the story, and the other is based on the experience of science and technology. Immersive entertainment projects based on plots are represented by immersive theatre, and Sleep No More is a well-known example. The audience's experience and the relationship between watching and acting have changed.[2] Moreover, there are other forms such as immersive room escape, role-playing game and spicy game event, setting the relationship between story lines and characters, and restoring the real scene.[3] The immersive entertainment projects focusing on the experience of science and technology are represented by the immersive exhibition. The exhibition contents are presented by means of science and technology to create a sense of experience in a large space, which can achieve the audience's active cognition of art works by the new media art form.[4] According to the concept and development of immersive entertainment IP experience project, combined with a variety of classification methods and characteristics of different immersion formats, it is found that immersive theatre, immersive room escape and immersive exhibition are the current mainstream forms with a certain scale and quantity, and have more advantages in form innovation and IP value embodiment. Therefore, this paper selects immersion theatre, immersive room escape and immersive exhibition as the key research.

The core of immersive entertainment IP experience project is IP. From the perspective of IP, there are two main shaping forms: original and adaptation. Original IP is easier to produce independent value and has more space for original play. For example, teamLab in Japan is the original IP representative of immersive exhibition, and the Mermaid by the dead water is the first original immersive theatre in China. In contrast, IP adaptation is a more mature and widely used IP creation mode in immersive entertainment IP experience projects. Immersive entertainment IP was born in the era of business fusion, with inherent conditions of integration.[5] It combines cross-border with science and technology, culture and art, expands the field of immersive entertainment IP, and introduces high-quality head IP of novels, animation, film and video games so as to help immersive entertainment projects enter the market quickly and trigger the emotional economy of immersive entertainment projects. The original value of IP has also been verified again, realizing the inheritance of its value, which has a crucial influence on immersive entertainment projects and IP itself.
Due to the late development of immersive entertainment project, its concept and application mode are not mature. It is worth thinking about the problems such as immersive chaos, insufficient communication, and the IP of immersive entertainment is not easy to be mined. There are few researches on immersive entertainment and its IP culture influence in the academic circle. However, some scholars at home and abroad mainly focus on digital technology, news communication, education and teaching, and they regard immersion as an auxiliary tool or means of communication to improve efficiency. In recent years, with the trend of cultural and technological integration, the immersive experience is studied as a new form of cultural industry, which would provide the new mechanism perspective and novel research angle.[6] In addition, in relevant research on cultural influence, Zhang et al.[7] evaluated the influence of new media information dissemination from the perspective of super IP. Zhou et al.[8] emphasized that in terms of cultural information, two important indicators that can measure the influence are the number of users and the time of users. Building on results of Guan's study,[9] the evaluation content of cultural international influence can be divided into three parts: the materialized form of culture (including cultural symbols and cultural products), the spiritual core of culture (including values, ways of thinking, and beliefs) and the communication channels of culture (including the public and outstanding people, cultural groups/organizations, and mass media).

In conclusion, there is no mature research model on the cultural influence of immersion entertainment IP experience project, which provides a great space for this paper and innovation approach. Thus, based on the influence research theory and the concept and characteristics of immersive entertainment IP experience project, this paper analyzes the factors of cultural influence of immersive entertainment IP experience project from the perspective of cultural industry development, constructs appropriate cultural influence model, and makes comprehensive empirical research from qualitative and quantitative perspective by using questionnaire survey and regression analysis. Finally, the paper will draw conclusions and provide reasonable and effective suggestions for the future development of immersive entertainment IP experience project.

\section{RESEARCH DESIGN}

For the immersive entertainment IP experience project, its cultural influence refers to the influence strength and effect of cognition, attitude and acceptance behaviour of IP experience project from the cultural level in the process of communication, which shows the depth and range of cultural identity of IP experience 
project. The realization of cultural influence is inseparable from the project itself and communication ability, and there is a certain logical relationship between these two parts and cultural influence, which cannot be replaced by each other. The formation of cultural influence is a dynamic process. In the immersive entertainment IP experience project, as an important cultural resource and foundation, IP experience project produces its cultural influence through the role of communication ability.

Therefore, the paper argues that the cultural influence of immersive entertainment IP experience project is mainly from five dimensions: form and packaging of project, IP value, service experience, dissemination of project and word of mouth. Among them, form and packaging of project, IP value and service experience are from the perspective of the project itself, explaining the role of the project itself for cultural influence from three aspects, while the project dissemination and word of mouth are from the perspective of communication ability, respectively reflecting the effect of communication channels and communication results on cultural influence. From the above five dimensions, we can have a comprehensive display of cultural influence analysis, and the relationship model is shown in Figure 1.

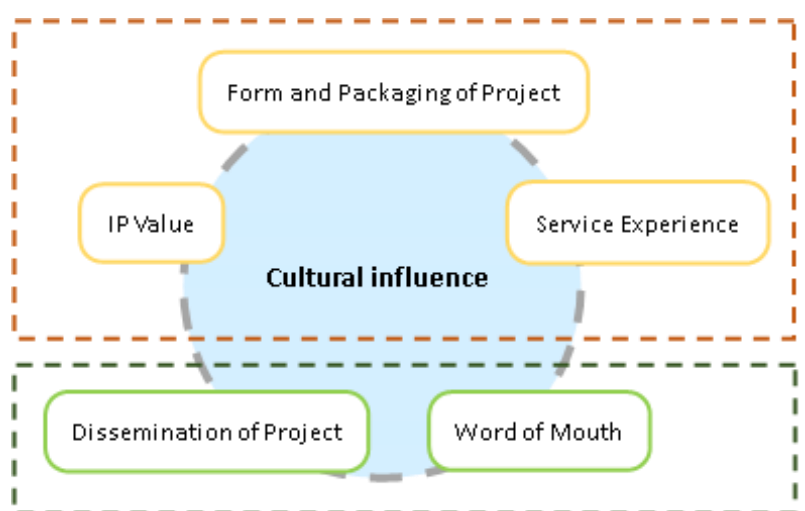

Figure 1 Research Model of Cultural Influence of

Immersive Entertainment IP Experience Project.

The form and packaging of project refers to the presentation form and external image of immersive entertainment IP experience project. IP value refers to the theme and content core of immersive entertainment IP experience project. Service experience, the added value brought by the immersive entertainment IP experience project, is mainly manifested in the service experience received by the participants in the whole process of project experience, including service scope and service attitude. The word of mouth refers to the praise and title of immersive entertainment IP experience project. Its reputation not only has the public oral evaluation, but also has the status and recognition in the society. Besides, the dissemination of project, namely the communication ability of immersive entertainment IP experience project, is reflected in the aspects of communication motivation, mode, scope and efficiency.

The paper uses a questionnaire survey to measure the cultural influence of immersive entertainment IP experience project. As the immersive entertainment IP experience project is mainly loved by the younger generation, the age range of the respondents is set at 2035 years old, which makes the survey results more representative. The contents of the questionnaire include the basic characteristics of the respondents, project participation, and attitudes towards the role of cultural influence in the above five dimensions. Among them, each dimension is set with 3-5 statements, with a total of 20 statements. The scale is composed of 20 statements from five dimensions. The respondents need to score and evaluate the statements according to their own situation, and the score range is set to an integer between 1 and 10 .

\section{RESEARCH ANALYSIS}

A total of 224 valid questionnaires were received in the study, and the effective recovery rate was $94.51 \%$. The data type of this article is cross-sectional data. The five dimensions of form and packaging of $\operatorname{project}\left(\mathrm{X}_{1}\right)$, IP value $\left(X_{2}\right)$, service experience $\left(X_{3}\right)$, word of mouth $\left(\mathrm{X}_{4}\right)$ and dissemination of project $\left(\mathrm{X}_{5}\right)$ are used as explanatory variables. Cultural influence(Y) is a multiple regression analysis for the explained variable, and the following equation is established:

$\mathrm{Y}_{\mathrm{i}}=\beta_{0}+\beta_{1} \mathrm{X}_{1 \mathrm{i}}+\beta_{2} \mathrm{X}_{2 \mathrm{i}}+\beta_{3} \mathrm{X}_{3 \mathrm{i}}+\beta_{4} \mathrm{X}_{4 \mathrm{i}}+\beta_{5} \mathrm{X}_{5 \mathrm{i}}+\mu_{\mathrm{i}}, \mathrm{i}=1,2, \ldots$ 224 , representing the number of valid questionnaires, $\beta_{0}$, $\beta_{1}, \beta_{2}, \beta_{3}, \beta_{4}$ and $\beta_{5}$ are six unknown parameters, and $\mu_{\mathrm{i}}$ is a random disturbance item.

The data of the explanatory variables are derived from the scores in the questionnaire and averaged to obtain five sets of data. The data of the explanatory variables are the overall scores of the respondents on the cultural influence in the questionnaire. Using EViews for regression analysis, the results are shown in Table 1.

Table 1. Regression Results of Cultural Influence

\begin{tabular}{|c|c|c|c|c|}
\hline Variable & Coefficient & Std. Error & t-Statistic & Prob. \\
\hline $\mathrm{X}_{1}$ & 0.164757 & 0.087539 & 1.882097 & 0.0216 \\
\hline$\overline{X_{2}}$ & 0.040296 & 0.087227 & 0.461966 & 0.6446 \\
\hline $\mathrm{X}_{3}$ & 0.062818 & 0.073137 & 0.858911 & 0.3913 \\
\hline $\mathrm{X}_{4}$ & 0.875224 & 0.105465 & 8.298726 & 0.0000 \\
\hline $\mathrm{X}_{5}$ & 0.315614 & 0.065339 & 4.830414 & 0.0267 \\
\hline $\bar{C}$ & 0.782551 & 0.338126 & 2.314378 & 0.0216 \\
\hline $\mathrm{R}$-squared & 0.647976 & \multicolumn{2}{|c|}{ F-statistic } & 80.25515 \\
\hline $\begin{array}{l}\text { Adjusted } \\
\text { R-squared }\end{array}$ & 0.639902 & \multicolumn{2}{|c|}{ Prob(F-statistic) } & 0.0000 \\
\hline
\end{tabular}


The regression result is:

$\mathrm{Y}^{\wedge}=0.164757 \mathrm{X}_{1 \mathrm{i}}+0.040296 \mathrm{X}_{2 \mathrm{i}}+0.062818 \mathrm{X}_{3 \mathrm{i}}+0.8752$ $24 \mathrm{X}_{4 \mathrm{i}}+0.315614 \mathrm{X}_{5 \mathrm{i}}+0.782551, \mathrm{i}=1,2 \ldots 224$, indicating the number of valid questionnaires.

The coefficient of each variable represents the degree of influence of the variable on the cultural influence. According to Table 1, form and packaging of $\operatorname{project}\left(\mathrm{X}_{1}\right)$, word of mouth $\left(\mathrm{X}_{4}\right)$ and dissemination of $\operatorname{project}\left(\mathrm{X}_{5}\right)$ can have a significant positive effect on the cultural influence of the explained variable. IP value $\left(\mathrm{X}_{2}\right)$ and service experience $\left(\mathrm{X}_{3}\right)$ can also have a positive impact on cultural influence, but the effect is average.

The paper combines the respondents' judgments on the five dimensions and conducts a comprehensive analysis with the degree of effect, in order to explore the actual situation of the cultural influence of immersive entertainment IP experience projects. The scores given by respondents for each dimension were averaged, so as to form the respondents' evaluation of the status quo of immersive entertainment IP experience project in five dimensions, and summarized in Table 2.

Table 2. Average Results of the Five Dimensions

\begin{tabular}{|c|c|}
\hline Dimension & Average \\
\hline Form and Packaging of Project $\left(\mathrm{X}_{1}\right)$ & 7.01 \\
\hline IP Value $\left(\mathrm{X}_{2}\right)$ & 6.59 \\
\hline Service Experience $\left(\mathrm{X}_{3}\right)$ & 6.39 \\
\hline Word of Mouth $\left(\mathrm{X}_{4}\right)$ & 6.63 \\
\hline Dissemination of Project $\left(\mathrm{X}_{5}\right)$ & 5.86 \\
\hline
\end{tabular}

According to the survey respondents' evaluation and scoring of the five dimensions in Table 2, and the effect of the five dimensions on cultural influence obtained above, a matrix comprehensive analysis is carried out. The result is shown in Figure 2.

\section{RESEARCH RESULT}

\subsection{The Word of Mouth Plays a Leading Role in Cultural Influence}

According to data analysis, word of mouth of projects can have a tremendous effect on cultural influence and become an important factor in recognition. The overall evaluation of the word of mouth is relatively high, which has been well received by most people. The content and quantity of comments on the cultural and spiritual level reflect the cultural influence of the immersive entertainment IP experience project. It can be seen that the immersive entertainment
IP experience project has gained a voice in reflecting the public's aesthetic and cognitive attitudes, promoting social progress and demonstrating cultural confidence. People increasingly are willing to include participating in IP experience projects as one of their social methods. When they enjoy their leisure life, they use marvellous and interesting ways to get the knowledge of popular science and life attitude enlightenment, which would improve the quality of life and enhance cultural literacy imperceptibly.

\subsection{The Promotion of Project Dissemination to Cultural Influence Is Insufficient}

As an indispensable factor of cultural influence, the communication ability and effect of the project have not yet played a full boost. According to the survey respondents' scores on the development status of the five dimensions, project communication is considered to be the lowest. At present, it seems that the immersive entertainment IP experience project has several shortcomings in dissemination, which are mainly reflected in the limited communication strength, small communication range, and single form of communication, which also implies that the immersive entertainment IP experience project has not yet reached the level worthy of high attention. So, it shows that this aspect also needs to make effective use of the upward space to continuously improve, in order to match the project dissemination ability with the effect of its communication on cultural influence.

\subsection{The Form and Packaging of Project Will Be the Icing on the Cake for Cultural Influence}

The form and packaging of the immersive entertainment IP experience project has the highest score in the five dimensions, which has received general attention and recognition from the public. Although it is not the core of project, it can present a glorious effect to enhance cultural influence. For the form and packaging of project, the effect on cultural influence can be measured mainly from the degree of novelty, applicability and the effect of using cultural elements. With the help of the novel and unique form of immersion, the project is packaged from various aspects such as site construction, route design, and installation use, color selection, clothing style matching and so on to attract people's attention and allow participants to immerse themselves in the scene. Furthermore, people can feel the thoughts conveyed by the work personally, and discover the cultural heritage and cultural value of the project through their ears and eyes. 


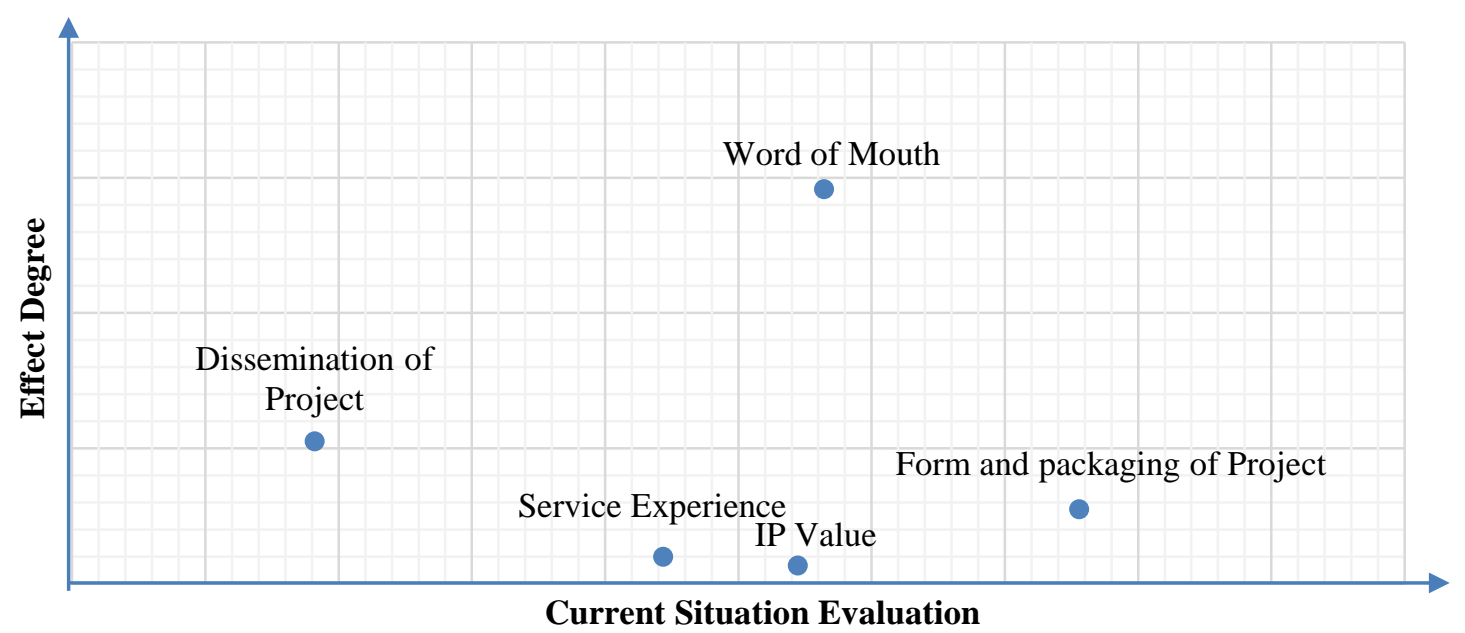

Figure 2 Comprehensive analysis of current situation evaluation and effect degree of five dimensions.

\subsection{The Effect of IP Value on Cultural Influence Needs to Be Further Improved}

As the core element of the cultural influence of the project, the IP value of the project is currently not obvious for its effect. The survey found that people's evaluation of the IP value is not satisfactory, and the value that can express cultural influence is very few, and the value of the cultural level is difficult to show. Fortunately, some immersive entertainment projects that are not refined enough will make the audience of the original IP feel that the IP is not respected, or the presentation form is too personalized to make it difficult to capture the connection between the project and the original IP. If those issues are not taken seriously, there may even be negative effects in the future, which will further affect the development and inheritance of IP value.

\subsection{The Immersive entertainment IP Experience Project Has Development Potential}

Immersive entertainment IP experience projects can be divided into three forms: immersive exhibition, immersive theatre and immersive room escape, and then compare the three types of public participation and cultural influence. The cultural influence is determined by the comprehensive score of the respondents on the cultural influence of the project and the respondents' willingness to participate in the project, that is, cultural influence $=$ comprehensive score $\times$ number of participant times / number of participants $\times 100 \%$. The results are shown in Figure 3.

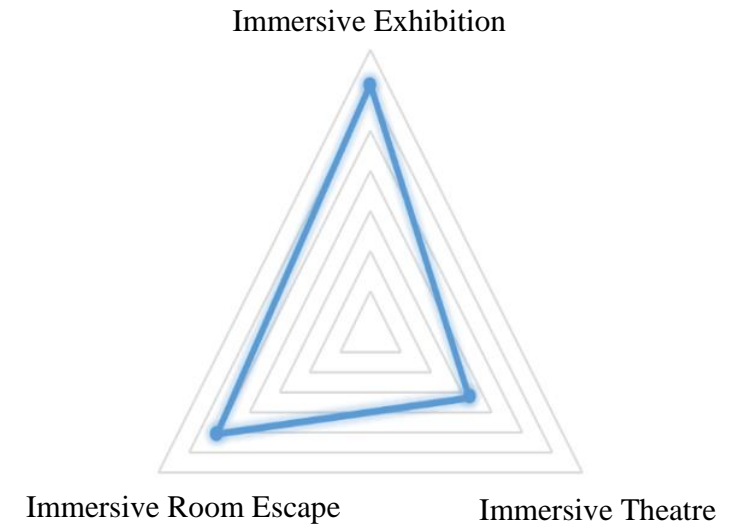

Figure 3 The Effect of three types of projects on cultural influence.

Among the three types of immersive entertainment IP experience projects, participants have the highest willingness for immersive room escape, followed by immersive exhibition, while their willingness to participate in immersive theatre is relatively low, which is almost proportional to the number and scale of projects. Immersive room escapes and immersive exhibitions can not only be set up in cultural and entertainment creative industry parks, but also in large shopping malls and supermarkets. They can be more easily noticed when they are set up in densely populated places, making the project more opportunities for participation and cultural influence. However, the immersive theatre has higher requirements for the venue. It usually requires a certain environmental area and complex building structure. Moreover, the time and number of immersive theatres will be subject to certain restrictions. In fact, immersive theatre also has its own advantages. Its content expression and the quality of works can play a role in cultural influence. Participants have the highest comprehensive scores for this type of project, and the IP value is more obvious. Therefore, the three mainstream forms of immersive entertainment IP 
experience projects each have their own characteristics, and create their own cultural influence from different modes and perspectives.

\section{CONCLUSION AND DEVELOPMENT SUGGESTION}

With the continuous development of immersive entertainment IP experience projects in recent years, the number of scales is increasing year by year, and the practitioners and audience groups are growing. The immersive entertainment IP experience projects keep up with the trend of the times; exert their fantastic ideas to create creative products with cultural influence, which has attracted more and more people's attention and pursuit. However, in the process of generally benign development, a series of problems have also emerged. The low popularity and repurchase rate of the project and the difficulty to reflect the cultural influence have become the bottleneck of the efficient development of the immersive entertainment IP experience project. In this regard, according to the current situation of immersive entertainment IP experience project, combined with the analysis results of its cultural influence, this paper puts forward suggestions for the sustainable development of immersion entertainment IP experience project.

\subsection{Improving the Overall Quality of the Project}

Because the immersive entertainment IP experience project is the output product of content creativity, it generally has the disadvantage of low repurchase rate, and the lack of high-quality projects. Thus, improving the quality of the project can attract repeat customers to improve the repurchase rate, and accumulate new customers to expand the popularity of the project. It can also fully reflect the energy and quality of IP, and enhance the connotation and cultural influence of the project.

From a macro point of view, the realization of highquality project development is for the people's livelihood, in order to provide more abundant cultural products, meet people's growing cultural needs, enhance people's cultural confidence, and realize high-level cultural consumption. In terms of specific suggestions, the development of immersive entertainment IP experience project needs superior and diversified teams, well-known IP licensing conditions and rich creativity, which can be fully supplied in material and spiritual resources, so as to lay a solid foundation for the production of high-quality works. In addition, there should be long-term IP shaping planning and content driven in terms of operation ideas, and the project can be set with multiple mainlines, so that all participants can unlock the whole project after many experiences, which can not only obtain higher participant flow, but also extend IP vitality.

\subsection{Creating a Project Integration Platform}

As far as the current situation is concerned, there is no relatively complete project system for immersive entertainment IP experience projects, and the content is scattered and difficult to control, which often leads to the buried of high-quality works for no reason and makes people lack of cognition or deviation on IP experience projects of immersion entertainment. Therefore, it is of great necessity to centralize the projects.

In the context of world connectivity, it is a remarkable strategy to create an offline store integration platform for immersive entertainment IP experience project, and is committed to creating a new platform with high-end service, high-quality integration and value-added empowerment. On the one hand, the establishment of a platform can centralize the project works to release and display the information of all kinds of immersive entertainment IP experience projects, set up a sharing space for the participants' experience of the project for exchange, and realize the project resource sharing. On the other hand, the platform can also become a means to strengthen the management of the whole system of immersive entertainment IP experience project.

\subsection{Deepening the Connection with Chinese Traditional Culture}

It is an effective way to enrich the cultural connotation of the project and improve the cultural influence of the immersion entertainment IP experience project. For one thing, sites with cultural basis can be selected to realize innovation. For example, immersive cultural and creative exhibitions are held regularly in museums and large bookstores, so as to change the old faces of those buildings, integrate projects and industries, and promote business innovation. For another, it is of significance to deeply develop IP with Chinese traditional culture connotation, and show the common sense of traditional culture through immersive entertainment IP experience project, such as adding some works related to festivals, creating immersive theatre with story situation based on the stories of festivals, creating immersive exhibition full of festival atmosphere, and setting puzzles and clues of immersive room escape around the festival.

\subsection{Enhancing Social Attention}

Combined with the results of the communication analysis of immersive entertainment IP experience project, it is considered that the immersive entertainment IP experience project needs to increase 
social attention in order to realize the wide spread of cultural influence and reflect the project value. For the head works with certain strength in the immersive entertainment IP experience project, the official media channels such as mass media and network media are used to publicize and obtain the basic recognition of the social immersion entertainment IP experience project. Besides, a number of internet celebrities and social celebrities in the cultural field are invited to view, personally participate in the experience and express their feelings to increase the popularity of the project with their traffic advantages. The immersive entertainment IP experience project is not a single business, but a display window of wisdom and IP.

\section{REFERENCES}

[1] Illuthion. The White Paper on the Development of China's Immersion Industry in 2020[EB/OL]. http://illuthion.com/talks/ciid2020whitepaper/ [2019.11.22] (in Chinese)

[2] Zhou Quan. Eastward Spread and Evolution: Chinese Immersive Theatre in Recent Years [J]. Drama (The Journal of the Central Academy of Drama), 2018(02):118-127. DOI: 10.13917/j.cnki. drama.2018.02.013 (in Chinese)

[3] Meituan Research Institute. 2019 China Room Escape Industry Consumption Insight Report [EB/OL]. https://mri.meituan.com/institute [2019. 12.5] (in Chinese)
[4] Chai Qiuxia. On Immersive Experience of Digital Media Interactive Art [J]. Art \& Design, 2012 (02):73-75. DOI: $\quad 10.16272 /$ j.cnki.cn111392/j.2012. 02.024 (in Chinese)

[5] Zhang Jun. Research on the Value Flow Rule of IP in Cultural Industry Chain [J]. Science-Technology \& Publication, 2017(01):104-108. DOI: 10.16510/j. cnki. kjycb.2017.01.026 (in Chinese)

[6] Hua Jian, Chen Qinghe. Immersive Experience: A New Business Format of the Integration of Culture and Technology [J]. Journal of Shanghai University of Finance and Economics, 2019, 21(05):18-32. DOI: 10.16538/j.cnki. jsufe. 2019.05.002 (in Chinese)

[7] Zhang Haitao, Zhang Huiran, Wei Ping, Li Tiyin. Construction of Evaluation Model of New Media Dissemination Influence in the Perspective of Super IP [J]. Information Science, 2019, 37(02):38. DOI: 10.13833/j.issn.1007-7634.2019.02.001 (in Chinese)

[8] Zhou Wen, Fan Xiaozhu. Discussion on the Possibility of Generating Chinese Cultural Influence under the Background of PanEntertainment [J]. Arts Criticism, 2018(02):62-67. DOI: $10.16364 /$ j.cnki.cn 11-4907/j.2018.02.010 (in Chinese)

[9] Guan Shijie. A Preliminary Study on the Evaluation System of International Influence of Chinese Culture $[\mathrm{J}]$. International Communications, 2015 (01):45-48. (in Chinese) 